\title{
Scalar mesons in a chiral theory with quark degrees of freedom
}

\author{
M. S. Lukashov and Yu. A. Simonov $\odot$ \\ Institute for Theoretical and Experimental Physics, NRC "Kurchatov Institute", Moscow 117218, Russia
}

(Received 16 October 2019; accepted 14 May 2020; published 26 May 2020)

\begin{abstract}
The chiral confining Lagrangian, based on chiral theory with quark degrees of freedom, is used to study the spectroscopy of scalar mesons. The formalism does not contain arbitrary fitting parameters, and it takes into account an infinite number of transitions from meson-meson to quark-antiquark states. Starting from known $q \bar{q}$ poles, the transition coefficients ensure the strong shift of the poles for $\pi \pi$ and a much smaller shift for the $K \bar{K}$ systems. The resulting amplitudes $f_{\pi \pi}$ and $f_{K \bar{K}}$ are calculated in terms of $q \bar{q}$ and the free meson Green's functions. Taking the $\pi \pi / K \bar{K}$ channel coupling into account, one obtains two resonances: a wide resonance $E_{1}$ in the range $500-700 \mathrm{MeV}$ and narrow $E_{2}$ near $1 \mathrm{GeV}$, which can be associated with $f_{0}(500)$ and $f_{0}(980)$. A similar analysis, applied to the $I=1$ channel, shows that in this case two very close poles in different sheets appear near $E=980 \mathrm{MeV}$, which can be associated with the $a_{0}(980)$ resonance. The obtained $\pi \pi$ interaction amplitudes, $\operatorname{Re} f_{\pi \pi}(E)$ and $\operatorname{Im} f_{\pi \pi}(E)$, are compared with the known data.
\end{abstract}

DOI: 10.1103/PhysRevD.101.094028

\section{INTRODUCTION}

Scalar mesons have been at the center of experimental and theoretical interest for a long time (see Ref. [1] for a summary of experimental data, the reviews [2-7] for information about the scalars, and [8,9] for a recent comprehensive analysis). The theoretical explanation of the scalar spectrum has faced difficulties and required the development of different approaches, like the tetraquark model [10], the molecular approach [11], and the QCD sum rules [12], as well as lattice calculations [13] (see the recent study [14]).

It is clear that in QCD any meson state can be represented as a series $M=c_{1}(q \bar{q})+c_{2}(q \bar{q})^{2}+\ldots$, where higher terms can be transformed into mesons as $(q \bar{q})^{n}=$ $m_{1} m_{2} \ldots$ Mesons with a nonzero $q \bar{q}$ component are called standard, while those with $c_{1}=0$ are called nonstandard or exotic. For standard mesons the original $q \bar{q}$ pole can be shifted due to the $q \bar{q}-m m-q \bar{q}$ interaction, as is known from comparison with experiment. However, the $m_{1} m_{2}$ interaction can be strong enough to produce bound states and resonances as in nuclear physics. In what follows we study scalar resonances in QCD, starting from the standard $q \bar{q}$ component and describing the physical scalar resonance as the result of multiple $q \bar{q}-m_{1} m_{2}$ transitions. In principle, this approach is not new and has been worked out

Published by the American Physical Society under the terms of the Creative Commons Attribution 4.0 International license. Further distribution of this work must maintain attribution to the author(s) and the published article's title, journal citation, and DOI. Funded by SCOAP ${ }^{3}$. in [15-22], where transitions have been properly parametrized. On the other hand, one can have additional poles due to the $m_{1} m_{2}$ interaction. The latter can be introduced in the framework of the meson-meson interaction in unitarized chiral perturbation theory (also on top of quark model resonances) [23-29], and here, e.g., $f_{0}(500)$ becomes heavier and more narrow when the $\mathrm{mm}$ interaction is suppressed $[24,25]$. For the results of unitarized chiral perturbation theory and inclusion of NLO terms, see $[27,28]$ and the review paper [8]. We stress that chiral perturbation theory is not necessary for obtaining these results, and one can use the dispersive methods and the data to obtain a good explanation of $f_{0}(500)$ and other resonances; see, e.g., Refs. [30-32].

The case of scalar mesons - and, in particular, lowest scalar mesons - is still unclear and further study is needed. As one can see in [1], Table 2, the conventional approach considers the resonances $a_{0}(1450)$ and $f_{0}(1370)$ as the lowest ${ }^{3} P_{0}$ states for $I=1,0$, respectively. On the other hand, numerous calculations of the lowest ${ }^{3} P_{0} q \bar{q}$ states with realistic $q \bar{q}$ interaction, including spin-dependent forces, refer to $a_{0}(980)\left[f_{0}(980)\right]$ as the lowest ${ }^{3} P_{0}$ states (see, e.g., Ref. [33]), while $a_{0}(1450)$ might only be connected to the first excited state.

There is no general consensus on the lowest states $\left[f_{0}(500), f_{0}(980), a_{0}(980)\right]$ in modern approaches, including the attempts to derive these states in molecular or tetraquark approaches. Unfortunately, in this latter approach, a recent lattice calculation [34] of the $a_{0}(980)$ state taking into account the tetraquark $\left(q^{2} \bar{q}^{2}\right)$ contribution does not show any explicit influence of the latter on the 
lowest states, thus calling for a new dynamics as a possible source of $f_{0}(500), f_{0}(980), a_{0}(980)$.

It is the purpose of the present paper to suggest a new approach to solve this problem and to demonstrate a new quark-chiral dynamics, which might explain the origin of the lowest scalar states. The essence of the method is as follows.

From our point of view, the main problem of most approaches to the scalar meson is the imbalance in the treatment of quark and meson degrees of freedom (d.o.f.). In reality, meson-meson $(\varphi \varphi$, e.g., $\pi \pi, K \bar{K})$ and $q \bar{q}$ d.o.f. have to be considered on equal footing since both can transform into each other at any moment in time. Moreover, the $q \bar{q}$ poles are accurately predicted at the proper places by relativistic QCD theory with scalar confinement and gluon exchanges [35-37] in all channels [38,39], and in many cases, they are observed in experiment shifted by $50-80 \mathrm{MeV}$ or less.

Therefore, the $q \bar{q}$ poles should be seen in experiment as the $\varphi \varphi$ resonances, shifted or not shifted. On the other hand, one may think of some (or all) $\varphi \varphi$ resonances as produced by the $\varphi \varphi$ interaction, e.g., by the unitarized chiral dynamics, where $q \bar{q}$ dynamics does not play any role. Instead, we consider the coupled $\varphi \varphi-q \bar{q}$ system with the proper $q \bar{q}$ dynamics and the transition dynamics of $q \bar{q}$ into the $\varphi \varphi$ system, first neglecting the $\varphi \varphi$ interaction and introducing it at the next stage.

Therefore, one needs the formalism of the two-channel $q \bar{q}, \varphi \varphi$ Green's functions, which takes into account any number of $q \bar{q}-\varphi \varphi$ and $\varphi \varphi-q \bar{q}$ transitions. In the case of scalar mesons, this type of formalism was already exploited in [15-22]. In the case of the heavy quarks, this formalism, considering the $Q \bar{Q}$ and $(Q \bar{q})+(\bar{Q} q)$ channels nonrelativistically, was suggested in [40], and it is called the Cornell formalism. It was used in [41] to discover the nature of the resonance $X(3872)$, with the $c \bar{c} 2^{3} P_{1}$ state transforming into $D D^{*}$ via a string breaking mechanism, which finally brings it to the $D_{0} D_{0}^{*}$ threshold at $3872 \mathrm{MeV}$ [42].

We generalize the Cornell formalism, making it relativistic and multichannel, when one $q \bar{q}$ state can transform into several $\varphi \varphi$ states. At the first stage, we neglect the interaction between white $\varphi \varphi$ mesons.

The full analysis of the scalars requires the multichannel approach to the problem, where several quark-antiquark $(q \bar{q})$ channels are present, together with two or more Nambu-Goldstone boson channels [ $(\varphi \varphi)$ channels]. Therefore, complete formulation requires the knowledge of (1) the Green's functions in both the $q \bar{q}$ and $\varphi \varphi$ channels and (2) the transition matrix elements between the channels. Without explicit knowledge of these entries, one faces the multiparameter and multichannel situation, where informative output is hardly possible.

The treatment of the first point-the spectral representation of the $q \bar{q}$ Green's function with accurate calculation of one-channel $q \bar{q}$ poles and couplings-can be done in the framework of the field correlator method (FCM) (see [36,37] for reviews and [38] for recent calculations in different channels). The $\varphi \varphi$ Green's function in the initial one-channel setup will be studied here, assuming that it can be replaced by the free two-body propagators and that resonances might exist due to channel coupling-in particular, with the $q \bar{q}$ channels. Here problem (2) becomes a basic point in a new approach.

Indeed, in heavy quarkonia the channel coupling with the heavy mesons is described by the string breaking mechanism (sometimes with emission of pions), which brings a resonance shift of $O(50-100) \mathrm{MeV})$. In the case of scalar mesons, the $\varphi \varphi$ channel contains chiral mesons, and the transition process from $\varphi \varphi$ to $q \bar{q}$ and back requires a different approach.

During the last 15 years, one of the authors Yu. S. has succeeded in deriving the chiral confining Lagrangian (CCL) - a powerful tool for the study of chiral effects in connection with quark d.o.f. $[43,44]$. The latter is actually an extension of the standard chiral Lagrangian, which contains both the quark and the chiral d.o.f. and tends to the standard chiral Lagrangian [45] when quark d.o.f. are neglected; all coefficients of CCL are easily calculated, as was done in [44] on the order of $p^{4}$. Moreover, the basic factors, like $f_{\pi}, f_{K}$, are calculated within this method [46]. The only basic parameter, $M(\lambda)=\sigma \lambda$, which appears due to confinement is a fixed quantity, defined by the transition radius $\lambda$. The latter is calculated at the stationary point and is expressed via string tension $\sigma$ and masses [47]; as a result, our method does not contain any fitting parameters. In this way the CCL method allows us to analytically find all entries for (1) and (2), while the scalar decay constants $f_{s}$ are calculated in the same way as $f_{\pi}, f_{K}$ within the FCM, using the spectral representation of the Green's function.

In principle, our method gives the possibility of treating any process with multiple $q \bar{q}$ and any number of $\varphi \varphi$ channels; the advantage of using the CCL is that for scalar mesons all transition coefficients are known. In the case of a single $\varphi \varphi$ and a single $q \bar{q}$ channel, our results can be written in the form comprising the Breit-Wigner resonance, similarly to results in Refs. [15-22]. However, in the case of multiple $\varphi \varphi$ channels, more complicated expressions are obtained, using the $K$-matrix approach.

As will be seen, the essence of our approach is the summation of the infinite rescattering series with multiple transitions between $\varphi \varphi$ and $q \bar{q}$ states, which yields several poles. In this way we obtain two poles in the regions of $f_{0}$ (500) and $f_{0}(980)$, which finally obtain realistic positions when the $\varphi \varphi$ interaction is taken into account.

The paper is organized as follows. In the next section the general structure of the coupled-channel Green's function for a scalar meson is derived from CCL, and we define basic quantities (1) and (2) in terms of known standard 
coefficients. In Sec. III we discuss the $q \bar{q}$ Green's functions in the spectral form and the free $\varphi \varphi$ Green's functions, and we use the decay constants and the pole masses from the known confining, gluon exchange, and spin-dependent interactions. Note that this calculation does not use any parameters, beyond the string tension, the current quark masses, $\Lambda_{\mathrm{QCD}}$, and $M(\lambda)$. In Sec. IV we discuss the resulting $\varphi \varphi \mid q \bar{q}$ Green's function and find the physical $\varphi \varphi$ amplitudes ( $\pi \pi$ and $K \bar{K}$ ), containing two resonances, which can be associated with $f_{0}(500)$ and $f_{0}(980)$. In Sec. V these results are augmented by the calculation of the real and imaginary parts of the $\pi \pi$ amplitude, which are qualitatively similar to the results obtained from theory and experiment, at least for $E>500 \mathrm{MeV}$. We demonstrate in Figs. 5 and 6 that, by a proper modification of the $\pi \pi$ Green's function, one is able to reproduce these data, and the resulting $\pi \pi$ resonance is closer to the experiment. We also show that, in the case of the isospin $I=1$, our method gives a different picture of two nearby poles within $50 \mathrm{MeV}$ in different sheets for $a_{0}(980)$. Section VI contains a discussion and an outlook.

\section{COUPLED-CHANNEL EQUATIONS FOR THE SCALARS FROM THE CHIRAL CONFINING LAGRANGIAN}

In what follows we use the CCL $[43,44]$ with the scalar external currents $s_{0}(x)$ and $s_{a}(x) \lambda^{a} \equiv \hat{s}$ for isospin $I=0$ and $I=1$, respectively:

$$
L_{C C L}=-N_{c} \operatorname{tr} \log \left(\hat{\partial}+\hat{m}+s_{0}+\hat{s}+M \hat{U}\right) .
$$

In Eq. (1) $\hat{\partial}$ implies $\frac{\partial}{\partial x_{\mu}} \gamma_{\mu}$, and $\hat{U}$ is the standard chiral operator,

$$
\begin{gathered}
\hat{U}=\exp \left(i \gamma_{5} \hat{\varphi}\right), \quad \hat{\varphi}=\frac{\varphi_{a} \lambda_{a}}{f_{a}}, \\
\hat{\varphi}=\sqrt{2}\left(\begin{array}{ccc}
\frac{1}{f_{\pi}}\left(\frac{\eta}{\sqrt{6}}+\frac{\pi^{0}}{\sqrt{2}}\right) & \frac{\pi^{+}}{f_{\pi}} & \frac{K^{+}}{f_{K}} \\
\frac{\pi^{-}}{f_{\pi}} & \left(\frac{\eta}{\sqrt{6}}-\frac{\pi^{0}}{\sqrt{2}}\right) \frac{1}{f_{\pi}} & \frac{K^{0}}{f_{K^{0}}} \\
\frac{K^{-}}{f_{K}} & \frac{\bar{K}^{0}}{f_{K^{0}}} & -\frac{2 \eta}{\sqrt{6} f_{\pi}}
\end{array}\right) .
\end{gathered}
$$

In (2) $\lambda_{a}$ are the Gell-Mann matrices, $\operatorname{tr} \lambda_{a} \lambda_{b}=2 \delta_{a b}$. One can consider CCL in (1) as a generating functional for different vertices and Green's functions. Indeed, omitting $s_{0}$ and $s$, one can expand, as in [43,44], in powers of $\hat{U}^{+} \Lambda(\hat{\partial}+\hat{m})(\hat{U}-1)=\eta$, which is dimensionless and yields an expansion in $S \hat{\partial} \hat{\phi}$, which is an expansion in quark loops (here $S=i \Lambda$ is the quark propagator) times the derivative of chiral field $\phi$. This gives $O\left(p^{4}\right)$ terms in good agreement with standard calculations, and the same expansion yields the standard GMOR relation-see [44] for details. In our case we need another expansion in powers of

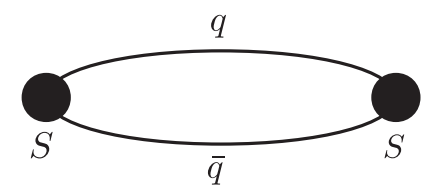

FIG. 1. The scalar $q \bar{q}$ Green's function $G_{q \bar{q}}$.

a dimensionless quantity, $\Lambda M(U-1)$, which is done as follows.

Using the scalar currents $s_{0}, \hat{s}$, one can generate the scalar Green's functions $G_{q \bar{q}}^{s}, G_{\varphi \varphi}^{s}$ :

$$
\begin{aligned}
L_{C C L} & =-N_{c} \operatorname{tr} \log \left(\Lambda^{-1}+s_{0}+\hat{s}+M(\hat{U}-1)\right) \\
& =-N_{c} \operatorname{tr} \log \Lambda^{-1}\left(1+\Lambda\left(s_{0}+\hat{s}+M(\hat{U}-1)\right)\right) \\
& =\frac{N_{c}}{2} \operatorname{tr}\left\{\left(\Lambda\left(s_{0}+\hat{s}\right) \Lambda\left(s_{0}+\hat{s}\right)\right)+\cdots\right\} \\
& =\frac{N_{c}}{2}\left(G_{q \bar{q}}^{s_{0}}+G_{q \bar{q}}^{\hat{s}}\right)+\cdots
\end{aligned}
$$

Here $\Lambda=\frac{1}{\hat{\partial}+\hat{m}+M}$. The corresponding diagram is shown in Fig. 1 One can write $G_{q}^{s}(x, y)=\operatorname{tr}\left(\bar{s}(x) g_{q \bar{q}}(x, y) s(y)\right)$, where $g_{q \bar{q}}$ will be used later.

On the other hand, expanding the CCL (4) in powers of $\Lambda M(\hat{U}-1) \equiv \xi$, one obtains another term in the second order in $\xi$,

$$
\Delta L=-N_{c} \operatorname{tr} \Lambda s \Lambda M \frac{\hat{\varphi}^{2}}{2}, \quad s=s_{0}+\hat{s},
$$

which corresponds to the diagram of Fig. 2. Note that in this way we can obtain the vertices for all chiral decays of any $q \bar{q}$ state, e.g., an $a_{2}$ meson decaying into $3 \pi$, etc.

In (1) the confining kernel $M(r)$ either enters the propagating $q \bar{q}$ system-in which case it is equal to the confining potential, $M(r)=\sigma r$-or it appears at the vertex of the $q \bar{q}$ Green's function, connecting it to the $\varphi \varphi$ Green's function. In this case the vertex $M(r)$ is taken at the effective distance $\lambda, M=M(\lambda)=\sigma \lambda$. One can consider this distance $\lambda$ as the spatial width of the transition vertex connecting $\varphi \varphi$ and $q \bar{q}$ channels (see Fig. 3). In the case of one chiral meson, we take it to be approximately equal to the correlation length in the confining vacuum, $\lambda \approx 0.2 \mathrm{fm}$, yielding $M(\lambda)=0.15 \mathrm{GeV}$. As a check of this approximation, this value was used to calculate $f_{\pi}$ and $f_{K}$ [46], in good agreement with experimental and lattice data;

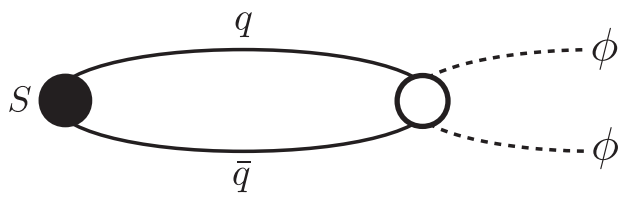

FIG. 2. The scalar $q \bar{q}$ Green's function with the emission of the chiral mesons. 


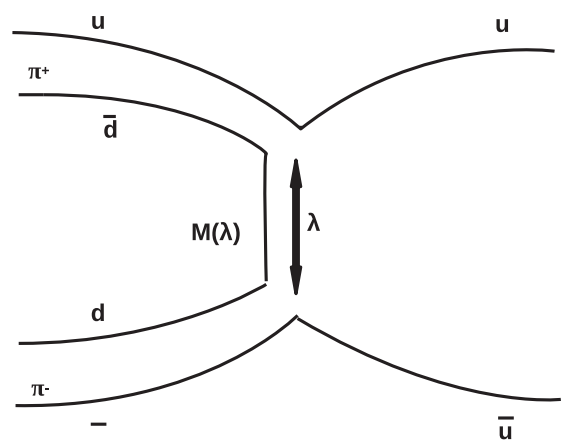

FIG. 3. The transition region $(q \bar{q} \mid \varphi \varphi)$ with the spatial distance $\lambda$ between the constituents.

therefore, we consider $\lambda$ in the range $(0.2-0.3) \mathrm{fm}$ (or $1-1.5 \mathrm{GeV}^{-1}$ ) in what follows. This factor $M(\lambda)$ appears to be the only parameter of our quark-chiral approach in (1), in addition to the quark masses $m_{q}$ and string tension $\sigma$.

From (5) one can find the basic quantity, which will be used below-the transition element $V_{q \bar{q} \varphi \varphi}$, which joins the $q \bar{q}$ Green's function $g_{q \bar{q}}$ and the $\varphi \varphi$ Green's function $g_{\varphi \varphi}$ (see Fig. 4 and its definition below). At this point it is important to understand which kind of $q \bar{q}$ Green's function is needed to join it with the $g_{\varphi \varphi}$, i.e., to annihilate at one vertex $q \bar{q}$ and create at this vertex two mesons $\varphi \varphi$. One clearly needs $g_{q \bar{q}}(x, y) \sim$ $\left(S_{q}(x, y) S_{\bar{q}}(x, y)\right.$, where $S_{q}(x, y)$ is the quark Green's function but with the definite total momentum, i.e., $g_{q \bar{q}}(P)=\int d^{4}(x-y) e^{i P(x-y)} \operatorname{tr}\left(S_{q}(x, y) S_{\bar{q}}(x, y)\right)$; $\quad$ originally, $g_{q \bar{q}}(x, y)$ should be connected with $g_{\varphi \varphi}$ at the same point $x$ or $y$ and finally with $g_{\varphi \varphi}(P)$. However, $g_{\varphi \varphi}(P)$ is divergent in its real part, which implies that the transition from $q \bar{q}$ to $\varphi \varphi$ occurs not at one point but at some distance between $q$ and $\bar{q}$, namely, at the same distance between $\varphi$ and $\varphi$, which we call $r_{0} \sim \lambda \sim 0.2 \mathrm{fm}$ - the transition radius, which is shown in Fig. 3.

It is important that at this moment the $M(r)$ becomes $M(\lambda)=\sigma \lambda \approx 0.15 \mathrm{GeV}$, and $\operatorname{Re} g_{\varphi \varphi}$ should have an initial and final $\varphi-\varphi$ distance $\lambda$. As will be shown below, this transition radius does not change the $g_{q \bar{q}}(\lambda)$ much, which is convergent at $\lambda=0$, but the variation of $\operatorname{Re} g_{\varphi \varphi}(\lambda)$ can be taken into account. In this approximation, the total scalar Green's function can be written as

$$
G^{s}=g_{q \bar{q}}^{s}+g_{q \bar{q}}^{s} V g_{\varphi \varphi}^{s} V g_{q \bar{q}}^{s}+\cdots=g_{q \bar{q}}^{s} \frac{1}{1-V g_{\varphi \varphi}^{s} V g_{q \bar{q}}^{s}} .
$$

Here $V \equiv V_{q \bar{q} / \varphi \varphi}$ can be found from (5) (see below).

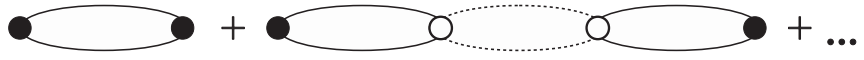

FIG. 4. The $\pi \pi$ interaction amplitude in terms of the $q \bar{q}$ (solid lines) and $\pi \pi$ Green's functions (broken lines). The filled and empty circles denote the transition matrix elements $V_{\pi 1}=V_{1 \pi}$.
As seen from (5), the transition coefficient $V$ is proportional to $\frac{M(\lambda)}{f_{\varphi}^{2}}, \varphi=\pi, K$, and also to the quark decay constant of the scalar meson $f_{s}^{(n)}(n=1,2, \ldots)$, as found below.

Finally, to define how $V$ depends on isotopic indices, one can, according to (5), project $\frac{\hat{\varphi}^{2}}{2}$ onto a given isotopic state with $I=0$ or 1 ,

$$
\operatorname{tr}\left(s_{0} \frac{\hat{\varphi}^{2}}{2}\right)=s_{0}\left(a_{11}+a_{22}+a_{33}\right)
$$

$$
\begin{aligned}
\operatorname{tr}\left(s_{i} \lambda_{i} \frac{\hat{\varphi}^{2}}{2}\right)= & a_{11}\left(s_{3}+\frac{1}{\sqrt{3}} s_{8}\right)+a_{22}\left(-s_{3}+\frac{1}{\sqrt{3}} s_{8}\right) \\
& +a_{12}\left(s_{1}+i s_{2}\right)+a_{21}\left(s_{1}-i s_{2}\right)-a_{33} \cdot \frac{2}{\sqrt{3}} s_{8},
\end{aligned}
$$

where $a_{i k}$ are

$$
\begin{gathered}
a_{11}=\frac{1}{f_{\pi}^{2}}\left[\left(\frac{\eta}{\sqrt{6}}+\frac{\pi^{0}}{\sqrt{2}}\right)^{2}+\pi^{+} \pi^{-}\right]+\frac{K^{+} K^{-}}{f_{K}^{2}}, \\
a_{12}=\frac{2 \eta \pi^{+}}{f_{\pi}^{2} \sqrt{6}}+\frac{K^{+} \bar{K}^{0}}{f_{K}^{2}}, \quad a_{21}=\frac{2 \eta \pi^{-}}{f_{\pi}^{2} \sqrt{6}}+\frac{K^{0} K^{-}}{f_{K}^{2}}, \\
a_{22}=\frac{1}{f_{\pi}^{2}}\left[\left(\frac{\eta}{\sqrt{6}}-\frac{\pi^{0}}{\sqrt{2}}\right)^{2}+\pi^{+} \pi^{-}\right]+\frac{K^{0} \bar{K}^{0}}{f_{K}^{2}}, \\
a_{33}=\frac{K^{+} K^{-}+K^{0} \bar{K}^{0}}{f_{K}^{2}}+\frac{2}{3} \frac{\eta^{2}}{f_{\pi}^{2}} .
\end{gathered}
$$

Later we shall neglect the isotopic [SU(3)] dependence of the propagators $\Lambda$, which is apparent in the mass matrices $\hat{m}$; we take it into account at the end since one can write $g_{q \bar{q}} \equiv g_{1}=\left(\begin{array}{cc}g_{1}(n \bar{n}) & 0 \\ 0 & g_{1}(s \bar{s})\end{array}\right)$.

\section{III. $q \bar{q}$ GREEN'S FUNCTIONS AND THE EIGENVALUES}

To calculate the $q \bar{q}$ Green's functions, we use the exact relativistic formalism, based on the FCM [35], essentially exploiting relativistic path integral methods [37-39,48,49]; at the end we compare our results with those obtained using other methods.

The $q \bar{q}$ Green's function $g_{q \bar{q}}^{\Gamma}(x, y) \equiv g_{1}(x, y)$ with the vertex $\Gamma$, defining the spin parity, can be written as

$$
g_{1}(x, y)=\operatorname{tr}\left(\frac{4 Y}{\left(m_{1}^{2}-\hat{D}_{1}^{2}\right)\left(m_{2}^{2}-\hat{D}_{2}^{2}\right)}\right)
$$

where 


$$
4 Y=\operatorname{tr}\left[\Gamma\left(m_{1}-\hat{D}_{1}\right) \Gamma\left(m_{2}-\hat{D}_{2}\right)\right]
$$

Then, using the relativistic path integral formalism (see [48-50] for a review), it can be written in the c.m. system and in the Euclidean time $T$,

$$
\begin{aligned}
& \int d^{3}(\mathbf{x}-\mathbf{y}) g_{1}(x, y) \\
& =\frac{T}{2 \pi} \int_{0}^{\infty} \frac{d \omega_{1}}{\omega_{1}^{3 / 2}} \int_{0}^{\infty} \frac{d \omega_{2}}{\omega_{2}^{3 / 2}}\langle Y\rangle\left\langle 0\left|e^{-H\left(\omega_{1}, \omega_{2}, \mathbf{p}\right) T}\right| 0\right\rangle .
\end{aligned}
$$

Here, the c.m. Hamiltonian $H\left(\omega_{1}, \omega_{2}, \mathbf{p}\right)$ depends on the virtual energies $\omega_{1}, \omega_{2}$ and includes all instantaneous interactions, including those that are spin and angular momentum dependent,

$$
H\left(\omega_{1}, \omega_{2}, \mathbf{p}\right)=\sum_{i=1,2} \frac{\mathbf{p}^{2}+\omega_{i}^{2}+m_{i}^{2}}{2 \omega_{i}}+V_{0}(r)+V_{\mathrm{so}}+V_{T} .
$$

Here, $V_{0}(r)=\sigma r-\frac{4}{3} \frac{\alpha_{V}(r)}{r}, V_{\text {so }}$ is the spin-orbit interaction, and $V_{T}$ is the tensor interaction, both in the relativistic form. Neglecting spin terms, one can rewrite the last term in (15) as

$$
\left\langle 0\left|e^{H\left(\omega_{1}, \omega_{2} \mathbf{p}\right) T}\right| 0\right\rangle=\sum_{n=0} \varphi_{n}^{2}(0) e^{-M_{n}\left(\omega_{1}, \omega_{2}\right) T},
$$

where $\varphi_{n}(\mathbf{r})$ is the wave function. On the other hand, one has a general relation

$$
\begin{aligned}
\int g_{1}(x, y) d^{3}(\mathbf{x}-\mathbf{y})= & \sum_{n} \int d^{3}(\mathbf{x}-\mathbf{y})\left\langle 0\left|j_{\Gamma}\right| n\right\rangle\left\langle n\left|j_{\Gamma}\right| 0\right\rangle \\
& \times e^{i \mathbf{P}(\mathbf{x}-\mathbf{y})-M_{n} T} \frac{d^{3} \mathbf{P}}{2 M_{n}(2 \pi)^{3}} \\
= & \sum_{n} \varepsilon_{\Gamma} \otimes \varepsilon_{\Gamma} \frac{\left(M_{n} f_{\Gamma}^{(n)}\right)^{2}}{2 M_{n}} e^{-M_{n} T} .
\end{aligned}
$$

This relation allows us to calculate the scalar decay constant $f_{s}^{(n)}$, which is done in Appendix A.

Note that using CCL [Eq. (1)], in (14) on obtains $m_{i}+M(\lambda)$ instead of $m_{i}$, which allows one to obtain, in the PS case $\left(\Gamma=\gamma_{5}\right)$, the correct decay constants $f_{\pi}, f_{K}$ [46], which otherwise would be zero in the zero quark mass limit.

In (15) it is convenient to integrate over $d \omega_{1}, d \omega_{2}$, using the stationary-point method, and for vanishing quark masses $m_{i}=0$, one obtains the so-called spinless Salpeter equation, if spin-dependent interactions are neglected. In the first approximation one has

$$
\left(2 \sqrt{\mathbf{p}^{2}}+V_{0}(r)\right) \varphi_{n l}(r)=M_{\operatorname{cog}}(n l) \varphi_{n l}(r)
$$

where $M_{\mathrm{cog}}$ means the center-of-gravity mass. Later we use only the fundamental parameters: $\sigma=0.182(2) \mathrm{GeV}^{2}$ and $\Lambda_{V}\left(n_{f}=3\right)=0.465(15) \mathrm{GeV}$, which are well established (see [51] for the definition of $\Lambda_{V}$ and an accurate perturbative treatment of scalar mesons); we obtain

$$
M_{\mathrm{cog}}(1 P)=1259(10) \mathrm{MeV}, \quad \omega_{0}(1 P)=499 \mathrm{MeV}
$$

Performing the same calculations as in [37-39], we give the resulting mass of the $1^{3} P_{0}$ state, taking into account the tensor and spin-orbit forces,

$$
M\left(1^{3} P_{0}\right)=(1259(10)-214) \mathrm{MeV}=1045(10) \mathrm{MeV}
$$

which defines the $q \bar{q}$ initial mass of $f_{0}$ and $a_{0}$, taken below, as $M_{1}=1 \mathrm{GeV}$. This mass can be compared with that obtained by other groups, where in [52] $M\left(0^{++}\right)=$ $1090 \mathrm{MeV}$, while in [53] $M\left(0^{++}\right)=1176 \mathrm{MeV}$, and in [54] $M\left(0^{++}\right)=970 \mathrm{MeV}$.

Note that the first excited state in the $0^{++}, I=0$ channel is obtained as $M_{2}=1474 \mathrm{MeV}$ [50], and this state can be associated with $a_{0}(1450)$.

Finally, we can use (18) to calculate the full Fourier transform of $g_{1}(x, y)$ in Minkowskian time, which yields

$$
\tilde{g}_{1}(P)=\tilde{g}_{1}(E, \mathbf{P}=0)=\sum_{n} \frac{\left(f_{s}^{(n)}\right)^{2} M_{n}^{2}}{M_{n}^{2}-E^{2}}
$$

The form with the lowest $n=1$ will be used below to analyze the scalars $f_{0}$; we show that the level $M_{1}=$ $1045 \mathrm{MeV}$ generates both $f_{0}(500)$ and $f_{0}(980)$ resonances, connected, respectively, to the $\pi \pi$ and $K \bar{K}$ Green's functions.

We now turn to the structure of the meson-meson Green's function, which we first take as a free two-body relativistic Green's function of two scalar particles with the total momentum $\mathbf{P}=0$ and the total c.m. energy $E$. See Appendix B for a detailed discussion.

Then, in the $\varphi \varphi$ channel, the free Green's function of $\varphi \varphi$ displaced by a spatial distance $\lambda$ and averaged over its direction ( $S$-wave) brings an additional factor $f_{2}(|\mathbf{p}| \lambda)$ :

$$
g_{2}(E)=\int \frac{f_{2}(|\mathbf{p}| \lambda) d^{4} p}{(2 \pi)^{4}\left(p^{2}-m_{1}^{2}\right)\left((P-p)^{2}-m_{2}^{2}\right)},
$$

with $\mathbf{P}=0 ; P_{0}=E$. Its imaginary part is 


$$
\operatorname{Im} g_{2}(E)=\frac{\sqrt{\left(E^{2}-\left(m_{1}+m_{2}\right)^{2}\right)\left(E^{2}-\left(m_{1}-m_{2}\right)^{2}\right)}}{16 \pi E^{2}} .
$$

One can compare (24) with the cutoff integral, where for equal masses $m_{1}=m_{2}=m$ one has, for the real part with the cutoff function $f_{1}(|\mathbf{p}| \lambda)=\theta(1-|\mathbf{p}| \lambda), N=1 / \lambda$,

$$
\operatorname{Re} g_{2}(E=2 m)=\frac{1}{8 \pi^{2}} \ln \left(\frac{N+\sqrt{N^{2}+m^{2}}}{m}\right) \text {. }
$$

Note that $f_{2}(|\mathbf{p}| \lambda)$ is not a cutoff introduced by hand, like $f_{1}(|\mathbf{p}| \lambda)$, which will be used below for comparison. Moreover, $f_{2}$ is part of a physical amplitude, not violating unitarity in the case of spatial distance and therefore not producing additional singularities. Indeed, one can see this in the explicit form since $f_{2}$ is expanded in even powers of $\mathbf{p}$.

In the case of the spatial cutoff $f_{2}(|\mathbf{p}| \lambda)$, when the initial and final distances between $\varphi \varphi$ are equal to $\lambda$, we have $f_{2}(x)=\left(\frac{\sin x}{x}\right)^{2}$, and the resulting difference between the two real parts with $f_{1}$ and $f_{2}$ is less than $10 \%$ for $\lambda=(0.5-2) \mathrm{GeV}^{-1}$.

Note that the spatial cutoff does not introduce branch points into $g_{2}(E)$ and therefore does not spoil unitarity.

\section{ANALYTIC STRUCTURE OF PHYSICAL AMPLITUDES}

We start with the transition coefficient, which we denote $k^{(I)}(q \bar{q} \mid \varphi \varphi)$, and define it in the following way. Using the definition of $\tilde{g}_{1}(P)$ in (22) and leaving for $\tilde{g}_{1}$ only the combination $\frac{M_{n}^{2}}{M_{n}^{2}-E^{2}}$, one can associate the transition coefficient with the following combination:

$$
\begin{aligned}
k^{(I)}(q \bar{q} \mid \varphi \varphi) & =V_{\bar{q} \bar{q} \mid \varphi \varphi} V_{\varphi \varphi \mid \bar{q} \bar{q}}=\left(V_{q \bar{q} \mid \varphi \varphi}\right)^{2} \\
& =\frac{C_{i}^{2} M^{2}(\lambda)\left(f_{s}^{(n)}\right)^{2}}{f_{\varphi}^{4}}, \quad f_{\varphi}=f_{\pi}, f_{K} .
\end{aligned}
$$

Here, the coefficient $C_{i}$ can be found from (7)-(12). Introducing the notation $C_{i}=C_{\text {meson,meson, one obtains, }}^{I}$ from (5) and (7)-(12),

$$
\begin{array}{ll}
\left(C_{\pi \pi}^{(0)}\right)^{2}=3, & \left(C_{K \bar{K}}^{(0)}\right)^{2}=2, \\
\left(C_{K \bar{K}}^{(1)}\right)^{2}=2, & \left(C_{\pi \eta}^{(1)}\right)^{2}=\frac{2}{\sqrt{3}} .
\end{array}
$$

We start with the one-threshold situation and choose the channel $\pi \pi$, neglecting its connection to $K \bar{K}$. In this case one has the following basic elements, with notation $g_{2}(\pi \pi, E) \equiv g_{\pi}, \quad \tilde{g}_{1}(E, \mathbf{P}=0)=g_{1}$, where we keep the lowest pole $M_{1}$, with the notation $V_{q \bar{q} \mid \pi \pi}=V_{1} \pi=V_{\pi 1}$,

$$
\begin{aligned}
k^{(0)}(n \bar{n} \mid \pi \pi) & =\left(V_{1} \pi\right)^{2}=\frac{\left(C_{\pi \pi}^{(0)}\right)^{2} M(\lambda)\left(f_{s}^{(1)}\right)^{2}}{f_{\pi}^{4}}=\left(V_{1} \pi\right)^{2}, \\
g_{1} & =\frac{M_{1}^{2}}{M_{1}^{2}-E^{2}},
\end{aligned}
$$

and the infinite series for the total $\pi \pi$ Green's function reads (see Fig. 3)

$$
G_{\pi \pi}=g_{\pi}+g_{\pi} V_{\pi 1} g_{1} V_{1 \pi} g_{\pi}+g_{\pi} V_{\pi 1} g_{1} V_{1 \pi} g_{\pi} V_{\pi 1} g_{1} V_{1 \pi} g_{\pi}+\cdots,
$$

which can be summed up in the form

$$
G_{\pi \pi}=g_{\pi}+g_{\pi} V_{\pi 1} \frac{1}{1-g_{1} V_{1 \pi} g_{\pi} V_{\pi 1}} g_{1} V_{1 \pi} g_{\pi} .
$$

For the $\pi \pi$ scattering amplitude $f_{\pi}(e)$, since $g_{\pi}$ does not contain $\pi \pi$ interaction, one can define

$$
G_{\pi \pi}=g_{\pi}+g_{\pi} f_{\pi}(E) g_{\pi},
$$

and one has

$$
f_{\pi}(E)=\frac{1}{16 \pi} V_{\pi 1} \frac{1}{1-\square_{\pi}} g_{1} V_{1 \pi},
$$

where we have defined the 4-term code $\square_{\pi} \equiv$ $g_{1} V_{1 \pi} g_{\pi} V_{\pi 1}=g_{1} g_{\pi} k^{(0)}(n \bar{n} \mid \pi \pi)$.

In an analogous way, one can define the one-channel $K \bar{K}$ Green's function and amplitude

$$
f_{K}(E)=\frac{1}{16 \pi} V_{K 1} \frac{1}{1-\square_{K}} g_{1} V_{1 K},
$$

where

$$
\begin{aligned}
\square_{K} & =g_{1} g_{K} k^{(0)}(n \bar{n} \mid K \bar{K}), \\
k^{(0)}(n \bar{n} \mid K \bar{K}) & =\left(\frac{C_{K \bar{K}}^{(0)} M(\lambda) f_{s}^{(1)}}{f_{K}}\right)^{2} .
\end{aligned}
$$

Note that the $q \bar{q}$ pole at $E^{2}=M_{1}^{2}$ is canceled in (31) and (32); the only visible singularity is the unitary cut in $g_{\pi}$ and $g_{K}$, respectively.

One can check the unitarity of both amplitudes $f_{\pi}$ and $f_{K}$,

$$
\operatorname{Im} f_{\pi}(E)=\frac{2 k_{\pi}}{E}\left|f_{\pi}(E)\right|^{2}
$$

and the similar form for $f_{K}$ is valid with the replacement $\pi \rightarrow K$.

One can also find the position of the pole in the amplitude $f_{\pi}(E)$ from the denominator in (31), $\square_{\pi}(E)=1$. One has 
$g_{\pi} k^{(0)}(n \bar{n} \mid \pi \pi) \frac{M_{1}^{2}}{M_{1}^{2}-E^{2}}=1, \quad g_{\pi}(E)=\operatorname{Re} g_{\pi}+i \operatorname{Im} g_{\pi}$.

Here, we take $M_{1}=1.05 \mathrm{GeV}$ as follows from (21).

In the real part of $g_{\pi}(E)$, the cutoff $N$ is taken at large momenta in (23), equal to the minimal length $\lambda, N=1 / \lambda$, which yields $(N=1 \mathrm{GeV})$,

$$
g_{\pi}(E) \approx 0.033+i 0.02 \sqrt{1-\frac{0.078}{E^{2}}} .
$$

Inserting in (35) for $I=0, f_{\pi}=93 \mathrm{MeV}, f_{s}^{(1)}=125 \mathrm{MeV}$ (see Appendix A for the discussion of $f_{s}^{1}$ and Appendix C for the pole position in the complex plane), and $M\left(\lambda=1 \mathrm{GeV}^{-1}\right)=180 \mathrm{MeV},\left(C_{\pi \pi}^{(0)}\right)^{2}=3$, one obtains the equation

$$
E^{2}=M_{1}^{2}\left(1-20.3 g_{\pi}(E)\right),
$$

or using (36), one obtains the resonance position $E_{\pi}=$ $(0.67-i 0.45) \mathrm{GeV}\left(\lambda=1 \mathrm{GeV}^{-1}\right)$. As a result, varying $\lambda$ in the range $(1-1.5) \mathrm{GeV}^{-1}$, one obtains the resonance parameters

$$
E_{\pi}=(0.6-0.8) \mathrm{GeV}-i(0.2-0.45) \mathrm{GeV} .
$$

Note that the resonance appears on the second sheet of the complex plane with respect to the $\pi \pi$ threshold, as it is explained in Appendix C.

This can be favorably compared with the experimental values $f_{0}(500), E=(400-550) \mathrm{MeV}, \Gamma=400-700 \mathrm{MeV}$ [1]. However, note that we have obtained these values with several simplifying approximations, including the neglect of higher levels in $g_{1}$, possible coupling with the $K \bar{K}$ channel, and notably neglecting the $4 \pi, 6 \pi, \ldots$ vertices of the chiral theory, which imply the $\pi \pi$ interaction in $g_{\pi}(E)$. Therefore, the resonance position and the width are subject to essential changes, if one takes this interaction into account. In particular, one can notice that the resonance position (38) is some (150-200) $\mathrm{MeV}$ higher than in experiment.

We now turn to the $K \bar{K}$ channel, again neglecting the connection to the $\pi \pi$ channel and keeping only the lowest mass eigenvalue $M_{1}=1.05 \mathrm{GeV}$ in $g_{1}(E)$. Inserting in (32) $\left(C_{K \bar{K}}^{(0)}\right)^{2}=2, f_{K}=115 \mathrm{MeV}, M\left(\lambda=1 \mathrm{GeV}^{-1}\right)=$ $180 \mathrm{MeV}$, and $f_{s}=125 \mathrm{MeV}$, one obtains $k^{(0)}(n \bar{n} K \bar{K})=$ 5.8. From (32) one finds the equation for the pole position, $\square_{K}=1$, or

$$
E^{2}=M_{1}^{2}\left(1-5.8 g_{K}(E)\right)
$$

where, with the upper limit $N=1 \mathrm{GeV}$ in (25),

$$
g_{K}(E)=0.018+i 0.02 \sqrt{\frac{E^{2}-4 m_{K}^{2}}{E^{2}}}
$$

which yields an approximate position of the pole,

$$
E_{K}=\left(\lambda=1 \mathrm{GeV}^{-1}\right)=(0.984-i 0.013) \mathrm{GeV} .
$$

One can see that the pole $E_{K}$ can be associated with the standard $f_{0}(980)$ [1],

$M\left(f_{0}(980)\right)=(990 \pm 20) \mathrm{MeV}, \quad \Gamma=(10-100) \mathrm{MeV}$,

while the obtained width is inside the allowed region. It is interesting that in this case the cutoff $\lambda$ in the range $(0.5-2) \mathrm{GeV}^{-1}$ brings about only a few percent change in the resulting resonance parameters. Taking into account the approximations made above, this agreement can be considered reasonable; however, one should take into account that both channels $\pi \pi$ and $K \bar{K}$ should be connected, as is seen in the experimental measurements of the ratio for $f_{0}(980), \frac{\Gamma(K \bar{K})}{\Gamma(\pi \pi)}=0.69 \pm 0.32[1]$.

The standard way to include the $\varphi \varphi$ channel coupling is to write, for the amplitudes $\hat{f}_{\alpha \beta}=\left(\begin{array}{c}f_{\pi \pi} f_{\pi K} \\ f_{K \pi} f_{K K}\end{array}\right)$, the $K$ matrix form,

$$
\begin{aligned}
\hat{f}^{-1} & =16 \pi\left(\begin{array}{ll}
\frac{1-\square_{\pi}}{w_{\pi}} & a \\
b & \frac{1-\square_{K}}{w_{K}}
\end{array}\right), \quad w_{\pi}=V_{\pi 1} g_{1} V_{1 \pi}, \\
w_{K} & =V_{k_{1} g_{1}} V_{i K} .
\end{aligned}
$$

As a result, one obtains

$$
\hat{f}=\frac{1}{16 \pi} \frac{\left(\begin{array}{cc}
\frac{1-\square_{K}}{w_{\pi}} & -b \\
-a & \frac{1-\square_{\pi}}{w_{K}}
\end{array}\right)}{\frac{1-\square_{\pi}}{w_{\pi}} \cdot \frac{1-\square_{K}}{w_{K}}-a b},
$$

and in the limit $a b=0$, one returns to the two independent channels.

One can check that the amplitudes $f_{\alpha \beta}, \alpha, \beta=\pi \pi, K \bar{K}$ satisfy the unitarity relations with the normalization factor $\operatorname{Im} g_{\pi, K}=\frac{k_{\pi, K}(E)}{8 \pi E}$. In particular, for $f_{K \bar{K}}$ one has, in this channel coupling (CC) form,

$$
16 \pi f_{K \bar{K}}=\frac{\left(1-\square_{\pi}\right) w_{K}}{\left(1-\square_{\pi}\right)\left(1-\square_{K}\right)-a b w_{K} w_{\pi}} .
$$

Estimating the $w_{K}, w_{\pi}$ one finds that the $\mathrm{CC}$ can affect the positions and the widths of the uncoupled resonances (38) and (41); therefore, this point should be studied in more detail. 
We start with the $f_{\pi \pi}$ amplitude, which can be written as follows:

$$
16 \pi f_{\pi \pi}=\frac{\frac{1}{w_{K}}-g_{K}}{\left(\frac{1}{w_{\pi}}-g_{\pi}\right)\left(\frac{1}{w_{K}}-g_{K}\right)-a b},
$$

and we can rewrite (46) using $\gamma=40.4 a b$ and the properly normalized amplitudes $\operatorname{Im} f_{\pi}^{(0)}=\frac{2 k}{E}\left|f_{\pi}^{(0)}\right|^{2}$ as follows:

$$
f_{\pi}^{(0)}=\frac{1}{\frac{16 \pi}{k} \frac{\left(E_{\pi}^{2}-E^{2}\right)}{M_{1}^{2}}-\frac{\gamma M_{1}^{2}}{E_{K}^{2}-E^{2}}}
$$

with

$$
\begin{aligned}
& E_{\pi}^{2}=M_{1}^{2}\left(1-k^{(0}(n \bar{n} \mid \pi \pi) g_{\pi}\right), \\
& E_{K}^{2}=M_{1}^{2}\left(1-k^{(0}(n \bar{n} \mid K \bar{K}) g_{K}\right) .
\end{aligned}
$$

Analogously for $f_{K}^{(0)}$ one has

$$
f_{K}^{(0)}=\frac{1}{\frac{16 \pi}{k M_{1}^{2}}\left(E_{K}^{2}-E^{2}\right)-\frac{2.34 \gamma M_{1}^{2}}{E_{\pi}^{2}-E^{2}}} .
$$

One can estimate the ratio of imaginary parts of the first and the second term in the denominator of (49), which yields the order of magnitude of the ratio of $\Gamma_{K \bar{K}}(f(980))$ and $\Gamma_{\pi \pi}(f(980))$ at $E=1.00 \mathrm{GeV}$,

$$
\frac{\Gamma_{\pi \pi}(f(980))}{\Gamma_{K \bar{K}}(f(980))} \cong \frac{2.4 \gamma \sqrt{E^{2}-4 m_{\pi}^{2}}}{\sqrt{E^{2}-4 m_{K}^{2}}} \approx 17 \gamma
$$

One can see that this ratio is around 1 for $\gamma=0.05$, which is found in the next section by comparison with data. The resulting pole is near the $K \bar{K}$ threshold and satisfies the criteria of the $f^{(0)}(980)$ resonance.

\section{RESULTS AND DISCUSSION}

From (47) one can see that the amplitude $f_{\pi}^{(0)}$ can be expressed via the Green's functions $g_{\pi}$ and $g_{K}$ with only the parameter $\gamma$ responsible for the coupling of channels $\pi \pi$ and $K \bar{K}$. Note that only the parameter $\lambda$ enters both $q \bar{q}-m m$ coupling $k^{(0)}$ and the real parts of both Green's functions, which start and finish at the same distance $\lambda$ and therefore contain the legitimate factor $N \sim 1 / \lambda \sim O(1 \mathrm{GeV})$. In the present paper we have chosen $\lambda$ in the narrow interval around $1 \mathrm{GeV}^{-1}$, which has yielded reasonable results. In a subsequent paper [47] it was shown that $\lambda$ can be defined from the stationary point of the transition coefficient $k^{(0)}$ and indeed has a value near $0.2 \mathrm{Fm}=1 \mathrm{GeV}^{-1}$.

The main result of our approach, based on the CCL (1), is that the $q \bar{q}$ pole at $1 \mathrm{GeV}$ can provide only one resonance, when connected with one threshold, and we need $\pi \pi-K \bar{K}$ channel coupling to produce two quarkchiral resonances: $f_{0}(500)$ due to coupling $n \bar{n}-\pi \pi$, and $f_{0}(980)$ due to coupling $n \bar{n}-K \bar{K}(n=u, d)$. This is a feature of our quark-chiral Lagrangian, and it is obtained from the infinite sum of products of $\square_{\pi}$ and $\square_{K}$. Starting with uncoupled $\pi$ and $K$ channels, it is interesting that the $\pi \pi$ pole, produced by the $q \bar{q}$ pole, is obtained without the $\pi \pi$ interaction, which is governed by the chiral Lagrangian; however, this occurs far from the experimental position, and then one needs direct (not via $q \bar{q}$ ) $\pi \pi$ interaction to bring resonance to the realistic values, which can be obtained directly by the analysis in [30-32]. The possible reason for this is that the low energy physics is only mildly connected to the higher $f^{(0)}$ resonance physics, but it strongly affects the low and intermediate energy regions, including the $f_{0}(500)$ position; at the first stage we have neglected the low energy $\pi \pi$ interaction given by the chiral Lagrangian.

Therefore, in our general two-channel form, we include a possible modification of the real and imaginary parts of $g_{\pi}$ due to direct $\pi \pi$ or $K \bar{K}$ interaction, which is contained in the term $E_{\pi}^{2}$ in (48). This leads to the following two-channel form, generalizing (47),

$$
\begin{gathered}
f_{\pi}=\frac{1}{2.67 \frac{\tilde{E}_{\pi}^{2}-E^{2}}{M_{1}^{2}}}-\frac{\gamma M_{1}^{2}}{E_{K}^{2}-E^{2}} ; \\
\tilde{E}_{\pi}^{2}=M_{1}^{2}\left(1-x(E)-i y(E) \sqrt{\frac{E^{2}-4 m_{\pi}^{2}}{E^{2}}}\right), \\
F_{2}(E)=0.96-0.043 i \sqrt{1-\frac{0.975}{E^{2}}} \theta\left(E^{2}-0.975\right)-E^{2} .
\end{gathered}
$$

Here, $x(E)=k \operatorname{Re} g_{\pi}(E), y=k \operatorname{Im} g_{\pi}(E)$ are found by fitting the resulting curves of $\operatorname{Re} f_{\pi}^{(0)}, \operatorname{Im} f_{\pi}^{(0)}$ to the data of [31,32]. Parameters of $x(E), y(E)$ are given in Appendix C.

The two curves $f_{\pi}(E)$, obtained by fitting $g_{\pi}(E)$, are shown in Figs. 4 and 5 by the solid lines, together with the curves from the work of Pelaez et al. [31], obtained in the course of the analysis in [32]. In the same figures we show the dashed lines obtained from (47) and (48) with the free $\pi \pi$ Green's function. As one can see, our $\operatorname{Re} f_{\pi}^{(0)}(E)$ and $\operatorname{Im} f_{\pi}^{(0)}(E)$ for the free case are in qualitative agreement with the results of [31], with an exclusion of the region of relatively small energies, $E<0.5 \mathrm{GeV}$.

This means that the $\pi \pi$ interaction is important in this region, and the approximation of the free $\pi \pi$ Green's function should be modified by inclusion of the purely chiral interactions, at least for the lowest $f_{0}(500)$ resonance.

This is well illustrated by the calculation of the position of the resonance $f_{0}(500)$, which was obtained above in the 


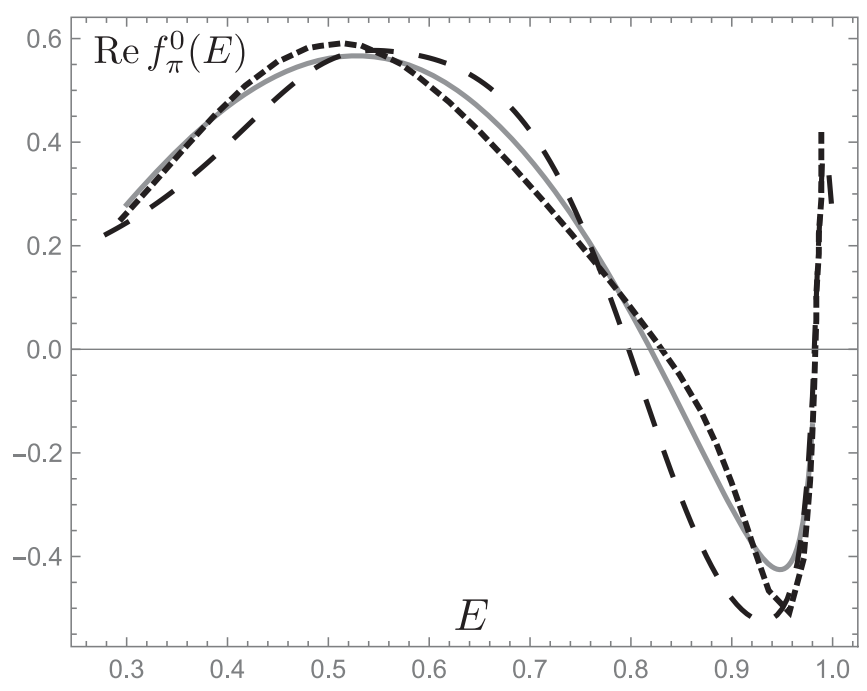

FIG. 5. $\operatorname{Re} f_{\pi}^{(0)}(E)$ as a function of $E$ in $\mathrm{GeV}$ from Eq. (47) (grey bands) in comparison with the resulting curves from Pelaez et al. [31,32] (broken lines) comprising the $\pi \pi$ data.

free $g_{\pi}$ case at $(0.67-i 0.45) \mathrm{GeV}$, while from [31] (the dotted lines in Figs. 5 and 6 ) the resonance position is at $(0.457-i 0.259) \mathrm{GeV}$.

However, it was not the purpose of our study to exactly reproduce the $\pi \pi$ interaction amplitude in the whole region (280, 1000) $\mathrm{MeV}$ but rather to discover the dynamical mechanism producing the lowest scalar-isoscalar mesons $f_{0}(500)$ and $f_{0}(980)$. At the first stage the basic part of this mechanism can be reduced to the interaction of the $q \bar{q}$ and free meson-meson channels, given by our quark-chiral interaction in the CCL, Eq. (1). Indeed, this interaction provides the reasonable coupling $V_{q \bar{q} \mid \pi \pi}$ and $V_{q \bar{q} \mid K \bar{K}}$, in addition to the values of the $q \bar{q}$ Green's functions and the corresponding poles $M_{n}(q \bar{q})$. In our case the lowest pole

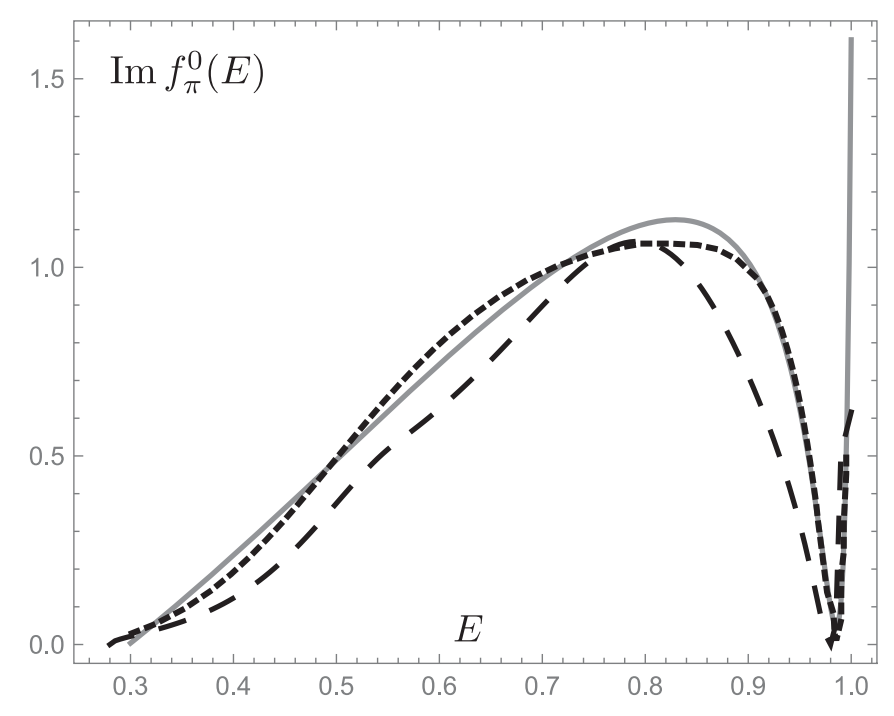

FIG. 6. The same as in Fig. 4 but for $\operatorname{Im} f_{\pi}^{(0)}(E)$.
$M_{1}(q \bar{q})$ at $1 \mathrm{GeV}$ produces a wide resonance $f_{0}(500)$ in "collaboration" with the $\pi \pi$ Green's function and the $\pi \pi$ threshold, and a more narrow resonance $f_{0}(980)$ in collaboration with the $K \bar{K}$ Green's function and the threshold. The interaction of these two channels, strongly shifted in energy from each other, which is outside of our simple $q \bar{q}$-meson-meson model, only slightly modifies their individual properties, as can be seen by comparing one-channel and coupled-channel characteristics.

In the second stage one should take into account the chiral interactions $(\pi \pi, K \bar{K})$ to obtain the relativistic $\pi \pi$ and $K \bar{K}$ amplitudes. This stage is especially essential for the determination of the $f_{0}(500)$ pole parameters, as can be seen from the analysis in [31]; $E=$ $(457 \pm 10) \mathrm{MeV}-i 279 \mathrm{MeV}$, which agrees with the results of [30]. This result disagrees with our estimate (38), where the $\pi \pi$ interaction was disregarded. At the same time the characteristics of the $f_{0}(980)$ in [31] and in our case, Eq. (41), are similar. This leads to the conclusion that the accurate determination of the lowest resonances, much below $1 \mathrm{GeV}$, requires a proper account of the $\varphi \varphi$ interaction, which can be done by combining the formalism of [30,31] with our $q \bar{q}-\varphi \varphi$ approach.

This is the main result of this paper; however, the general mechanism described above leads to many further possible discoveries.

At this point one can ask the following question: If the same $q \bar{q}$ level can create several resonances, accounting for the coupling between $\varphi \varphi$ channels, what happens with the $a_{0}(980)$ resonance, which can decay both to $\pi \eta$ and $K \bar{K}$ ? However, in experiment one can see only one broad resonance near the $K \bar{K}$ threshold. Now we apply our technique to this case to understand the difference between the situation with $a_{0}(980)$ on one hand and $f_{0}(500)$, $f_{0}(980)$ on the other.

To this end we try to find separate resonances in the $\pi \eta$ and $K \bar{K}$ channels and write, as in (37), the resulting equation for the position of the assumed resonances $E^{(1)}(\pi \eta)$ and $E^{(1)}(K \bar{K})$, where the upper index refers to the isospin $I=1$ :

$$
\left(E^{(1)}(\nu)\right)^{2}=M_{1}^{2}\left(1-k^{(1)}(n \bar{n} \mid \nu) g_{\nu}(E)\right) ; \quad \nu=\pi \eta, \quad K \bar{K} .
$$

Now, using (28) and (8)-(12) one can write

$$
\begin{gathered}
k^{(1)}(n \bar{n} \mid \pi \eta) \approx V_{\pi \eta, 1} V_{1, \pi \eta}=\left(C_{\pi \eta}^{(1)}\right)^{2} \frac{M^{2}(\lambda)\left(f_{s}^{(1)}\right)^{2}}{f_{\pi}^{2} f_{\eta}^{2}}=4.69, \\
k^{(1)}(n \bar{n} \mid K \bar{K})=\frac{\left(C_{K \bar{K}}^{(1)}\right)^{2} M^{2}(\lambda)\left(f_{s}^{(1)}\right)^{2}}{f_{K}^{4}}=2.9 .
\end{gathered}
$$


[We have neglected the difference between $f_{\pi}$ and $f_{\eta}$ for a rough estimate; in the real case the coefficient in (54) is smaller.]

Now, $g_{\pi \eta}(E)$ has smaller real and imaginary parts (see Appendix B for details) as compared with $g_{\pi \pi}(E)$, Eq. (36), while $g_{K \bar{K}}(E)$ is the same as was used before [see Eq. (40)]. As a result, the solution of Eq. (53) gives two resonances:

$$
E^{(1)}(\nu)=M_{1}\left(1-\bar{a}_{\nu}-i \bar{b}_{\nu}\right)
$$

where a rough estimate yields

$$
\begin{gathered}
\bar{a}_{\pi \eta} \cong 0.05, \quad \bar{b}_{\pi \eta} \approx 0.05, \\
\bar{a}_{K \bar{K}}=0.022, \quad \bar{b}_{K \bar{K}}=0.04 \sqrt{\frac{E^{2}-4 m_{K}^{2}}{E^{2}}} .
\end{gathered}
$$

One should take into account that $M_{1}(I=1) \approx M_{1}(I=0)=$ $1.00 \mathrm{GeV}$ and $E^{(1)}(\pi \eta) \cong(1.05) \mathrm{GeV}$, while $E^{(1)}(K \bar{K}) \cong$ $\left(1.04-i 0.02 \sqrt{\frac{E^{2}-4 m_{k}^{2}}{E^{2}}}\right) \mathrm{GeV} ; E^{(1)}(\pi \eta)$ is on the second sheet with the $\pi \eta$ threshold, while $E^{(1)}(K \bar{K})$ is on the second sheet with the $K \bar{K}$ threshold.

However, one should consider the $\pi \eta-K \bar{K}$ channel coupling, which can rearrange the position of the poles, as was found recently in the lattice calculations [14].

Thus, one can see that the displacements of both resonances are small, being of the order of the width of the resonances. This might be the reason why in experiment one actually observes one resonance $a_{0}(980)$ near $1 \mathrm{GeV}$ with two decay modes, while in the $I=0$ channel with larger couplings $k^{(0)}(n \bar{n} \mid \pi \pi)$ and more distant $\pi \pi$ and $K \bar{K}$ thresholds, one observes two distinct resonances; this example gives additional support for our theory.

\section{CONCLUSIONS AND OUTLOOK}

Comparing our approach with other models, we neglect any direct $\varphi \varphi$ interaction in the first step described in the paper. Therefore, all details of this interaction, as well as the $q \bar{q}-q \bar{q}$ interaction-in particular, crossing symmetry, the left-cut singularities, etc.- - are missing in this first step. As a second, more complicated step, one should take $\mathrm{i}$ nto account all the details of the $\varphi \varphi$ interaction, e.g., as in the dispersive methods or in unitarized chiral model interaction.

In summary, the method suggested above as a first stage has a general character and can be applied to any systems, consisting of several components, which can transform into one another. The only information needed to describe the properties of such mixed systems are the spectral properties of each component and transition coefficients. In the case of the charmonium system, this method gives a first explanation of the resonance $X(3872)$ [42]. In the case of the quark-chiral system, $q \bar{q}-\varphi \varphi$, this method uses the information given by the FCM approach plus the quarkchiral CCL Lagrangian (1). Our method suggests a possible solution to the old-standing problem of $f_{0}(500), f_{0}(980)$, and $a_{0}(980)$ associating these resonances with $(n=1) q \bar{q}$ ${ }^{3} P_{0}$ states.

As applied to the lowest scalar resonances, we have shown that the resonances $f_{0}(500)$ and $f_{0}(980)$, as well as the $a_{0}(980)$ resonance, can be connected to the use of CCL and to the $n=1, M \approx 1 \mathrm{GeV} q \bar{q}$ resonance, calculating explicitly the transition coefficients and, consequently, the partial widths. Several issues arise:

(1) Since we have connected $f_{0}(500), f_{0}(980)$ with one $q \bar{q}$ state- the ${ }^{3} P_{0}$ ground state $n \bar{n}$ with mass around $1 \mathrm{GeV}$-one should consider the next $q \bar{q}$ state, $M_{2}(1474)$, as an excited $q \bar{q}$ state with $n=2$, in contrast to an accepted view (see [1]) that the latter is a ground state. It is interesting to study the consequences of this assignment.

(2) What will the result be for excited $q \bar{q}-\varphi \varphi$ states, e.g., with $M_{2}=1474 \mathrm{MeV}$, in connection with the same $\varphi \varphi$ thresholds, and can one expect additional resonances below $M_{2}$ ?

(3) It is clear that by taking into account the full sum $\sum_{n} \frac{M_{n}^{2}}{M_{n}^{2}-E^{2}}$, one finds divergences and the necessity for renormalization. This issue can probably be treated in the spirit of the formalism developed in the method of matrix product states; see [55] for reviews.

(4) We have considered only one $q \bar{q}$ channel. However, for the $K \bar{K}$ system the $s \bar{s}$ channel provides bound states starting with $M_{1} \cong 1400 \mathrm{MeV}$, near the first excited $n \bar{n}$ state. Therefore, for the $K \bar{K}$ system one should take into account both $n \bar{n}$ and $s \bar{s}$ states, which requires an extension of our method with the inclusion of several $q \bar{q}$ and one or more $\varphi \varphi$ channels to explain several extra resonances in the region 1300-1700 MeV, observed in experiment [1].

These topics have recently been studied in [47], and it was shown how excited $q \bar{q}$ states produce higher scalar resonances using the explicit method discussed in the present paper. In addition, $n \bar{s}$ and $s \bar{s}$ systems have also been considered in connection with the corresponding $\mathrm{mm}$ thresholds, and the same pole shifts as in the present paper were found. From this point of view our approach helps clarify the old problem of scalar resonances, both in the ground and first excited states. The following question remains for future investigations: What parts of the lowest scalars are occupied by $q \bar{q}$ and direct $\varphi \varphi$ interactions?

\section{ACKNOWLEDGMENTS}

This work was completed in the framework of the scientific project supported by the Russian Science Foundation Grant No. 16-12-10414. The authors are indebted to A.M. Badalian for many discussions, 
suggestions, and details, which are used in the paper. Discussions with Yu.S. Kalashnikova and Z. V. Khaidukov are gratefully acknowledged.

\section{APPENDIX A: DECAY CONSTANTS OF SCALAR MESONS}

In the framework of the path-integral formalism, the decay constants of the $q \bar{q}$ meson states can be defined as in $[39,50]$,

$$
\left(f_{\Gamma}^{(n)}\right)^{2}=\frac{2 N_{c}\left\langle Y_{\Gamma}\right\rangle\left|\varphi_{n}(0)\right|^{2}}{\omega_{1} \omega_{2} M_{n}}
$$

where $\omega_{1}, \omega_{2}$ are the average energies of quarks with masses $m_{1}$ and $m_{2}, M_{n}$ is the mass of the meson, $\varphi_{n}(r)$ is the (relativistic) meson wave function of the relative distance $r$, and $\left\langle Y_{\Gamma}\right\rangle$ is

$$
\begin{aligned}
4 Y_{\Gamma} & =\operatorname{tr}\left(\left(m_{1}-\hat{D}_{1}\right) \Gamma\left(m_{2}-\hat{D}_{2}\right) \Gamma\right) \\
& =\operatorname{tr}\left(\left(m_{1}-i \hat{p}_{1}\right) \Gamma\left(m_{2}+i \hat{p}_{2}\right) \Gamma\right) .
\end{aligned}
$$

Here, $\Gamma$ is the vertex operator, for the scalar particle $\Gamma_{s}=1$, but the momentum operators $\hat{p}_{i}$ act on the wave function $\varphi_{n}(r)$, namely, $i p_{i} \varphi_{n}(r)=\partial_{i} \varphi_{n}(r)$. In our case

$$
\begin{aligned}
\left\langle Y_{s}\right\rangle\left|\varphi_{n}(0)\right|^{2}= & \left(m_{1} m_{2}-\omega_{1} \omega_{2}-\hat{\mathbf{p}} \hat{\mathbf{p}}^{\prime}\right)\left|\Psi_{S}(0)\right|^{2} \\
& \rightarrow\left(\partial_{i} \Psi_{S}(\mathbf{r}) \partial_{i}^{\prime} \Psi_{S}^{*}\left(\mathbf{r}^{\prime}\right)\right)_{r \rightarrow 0, r^{\prime} \rightarrow 0}
\end{aligned}
$$

Since $\Psi_{S}(\mathbf{r})$ is

$$
\Psi_{S}(\mathbf{r})=\sum \chi_{1 M_{1}} \tilde{Y}_{1 m_{2}} \frac{\varphi(r)}{r} C_{1 m_{1}, 1 m_{2}}^{00}
$$

and $\tilde{Y}_{1 m} \equiv r Y_{1 m}$, after summation over spin projections, one finds $\partial_{i} \Psi_{S}(\mathbf{r}) \partial_{i}^{\prime} \Psi_{S}^{*}\left(\mathbf{r}^{\prime}\right)=\partial_{i} \partial_{i}^{\prime} \frac{1}{4 \pi}\left(x x^{\prime}+y y^{\prime}+z z^{\prime}\right)=\frac{1}{4 \pi}$,

where we have taken into account that the subscript $i$ refers to a fixed momentum direction. As a result, one obtains

$$
\left(f_{S}^{(n)}\right)^{2}=\frac{2 N_{c}\left(R_{n P}^{\prime}(0)\right)^{2}}{4 \pi \omega_{1} \omega_{2} M_{n}}
$$

where $R_{n P}^{\prime}(0)=\left(\frac{\varphi_{n}(r)}{r}\right)_{r \rightarrow 0}$. Estimated in the same way as in $[39,50]$ for the $1 P$ scalar state, one has $R_{1 P}^{\prime}(0)=$ $0.086 \mathrm{GeV}^{5 / 2}, \omega_{1}=\omega_{2}=0.448 \mathrm{GeV}$ [56], and according to (A6) one obtains

$\left(f_{S}^{(1)}\right)^{2}=0.01568 \mathrm{GeV}^{2}, \quad f_{S}^{(1)}=0.125 \mathrm{GeV}$.

For the first excited state, $2 P$, one obtains, for the scalar state $\omega(2 P) \cong 0.5 \mathrm{GeV}, R_{2 P}^{\prime}(0)=0.0817 \mathrm{GeV}^{5 / 2}, M(2 P)=$ $1.474 \mathrm{GeV}$ [56].

As a result, from (A6) one obtains

$\left(f_{s}^{(2)}\right)^{2}=0.00865 \mathrm{GeV}^{2}, \quad f_{s}^{(2)}=0.093 \mathrm{GeV}$.

\section{APPENDIX B: MESON-MESON GREEN'S FUNCTIONS}

The relativistic Green's function of two scalar mesons with the total momentum $P$ can be written in the Euclidean space-time as

$$
g(P)=\int \frac{d^{4} p}{(2 \pi)^{4}} \frac{1}{\left[(P-p)^{2}+m_{1}^{2}\right]\left(p^{2}+m_{2}^{2}\right)} .
$$

Integrating over $d p_{4}$ in the c.m. frame, $\mathbf{P}=0$, one obtains, with $P_{4}=i E$ and $m_{1}>m_{2}$,

$\operatorname{Re} g_{12}(E)=\int_{0}^{N} \frac{p^{2} d p}{4 \pi^{2}} \times \frac{E\left(\sqrt{p^{2}+m_{1}^{2}}+\sqrt{p^{2}+m_{2}^{2}}\right)+m_{1}^{2}-m_{2}^{2}}{\sqrt{p^{2}+m_{1}^{2}} \sqrt{p^{2}+m_{2}^{2}}\left[\left(\sqrt{p^{2}+m_{1}^{2}}+\sqrt{p^{2}+m_{2}^{2}}\right)^{2}-E^{2}\right]\left(E+\sqrt{p^{2}+m_{1}^{2}}-\sqrt{p^{2}+m_{2}^{2}}\right)}$.

Here, we have introduced the cutoff $N$ in momentum $p$ :

$\operatorname{Im} g(E)=\frac{1}{16 \pi} \frac{\sqrt{\left(E^{2}-\left(m_{1}+m_{2}\right)^{2}\right)\left(E^{2}-\left(m_{1}-m_{2}\right)^{2}\right)}}{E}$.

In the equal mass limit one obtains
$\operatorname{Re} g(E)=\int_{0}^{N} \frac{p^{2} d p}{8 \pi^{2} \sqrt{p^{2}+m^{2}}\left(p^{2}+m^{2}-E^{2} / 4\right)}$,

which for $E^{2}=4 m^{2}$ reduces to a simple answer,

$\operatorname{Re} g(2 m)=\frac{1}{8 \pi^{2}} \int_{0}^{N} \frac{d p}{\sqrt{p^{2}+m^{2}}}=\frac{1}{8 \pi^{2}} \ln \frac{N+\sqrt{N^{2}+m^{2}}}{m}$. 
For $E^{2}=4 m^{2}-4 \Delta, \Delta>0$ one has, instead of (B5),

$$
\begin{aligned}
\operatorname{Re} g(E)= & \frac{1}{8 \pi^{2}} \int_{0}^{N} \frac{d p}{\sqrt{p^{2}+m^{2}}} \\
& -\frac{\Delta}{8 \pi^{2}} \int_{0}^{N} \frac{d p}{\sqrt{p^{2}+m^{2}}\left(p^{2}+\Delta\right)},
\end{aligned}
$$

and for $m^{2} \gg \Delta$ the last integral in (B6) can be written as

$$
\Delta \operatorname{Re} g(E) \cong-\frac{\sqrt{\Delta}}{16 \pi m} \theta\left(m^{2}-\frac{E^{2}}{4}\right)
$$

Note that $\Delta \operatorname{Re} g(E)$ is much smaller than $\operatorname{Re} g(2 m)$, Eq. (B5), and can be neglected in the first approximation.

\section{APPENDIX C: POSITION OF NEW POLES IN THE COMPLEX PLANE}

One can write Eq. (37) for the $\pi \pi$ pole as

$E^{2}=M_{1}^{2}\left(1-\operatorname{const}\left(\operatorname{Re} g_{\pi}(E)\right)+i \operatorname{const}\left(\operatorname{Im} g_{\pi}(E)\right)\right)$.

Writing $\operatorname{Im} g_{\pi}(E)=$ const $p(E) / E$, where $p(E)=$ $\sqrt{E^{2}-4 m_{\pi}^{2}}$, one can rewrite $(\mathrm{C} 1)$ as $E^{2}=E_{0}^{2}-\frac{i b p(E)}{E}$, or expressing $E^{2}$ via $p^{2}$, one has

$$
p^{2}(E)+\frac{i b p(E)}{\sqrt{p^{2}(E)+4 m_{\pi}^{2}}-p_{0}^{2}}=0
$$

where $b$ and $p_{0}$ are constants. Starting with small $b$, one gets approximately $p(E)=p_{0}$, and in the next order with $\sqrt{p^{2}(E)+4 m_{\pi}^{2}}=\sqrt{P_{0}^{2}+4 m_{\pi}^{2}}$, one solves the quadratic equation for $p(E)$ as

$$
\begin{aligned}
p(E)= & -\frac{i b}{2 \sqrt{p^{2}(E)+4 m_{\pi}^{2}}} \\
& \pm \sqrt{\frac{-b^{2}}{4\left(\sqrt{p^{2}(E)+4 m_{\pi}^{2}}\right)^{2}}+p_{0}^{2}} .
\end{aligned}
$$

One can perform the next orders of approximation following the motion of the root, starting with the position (C3). Another method is the direct solution of Eq. (C2), which is cubic in $p^{2}(E)$, choosing the correct root to be consistent with (C3). As is seen in (C3), the sign of the imaginary part of $p(E)$ is negative, implying that the pole is on the second sheet in the $E$ plane, corresponding to the $\pi \pi$ threshold.
[1] M. Tanabashi, K. Hagiwara, K. Hikasa et al. (Particle Data Group), Phys. Rev. D 98, 030001 (2018).

[2] F. E. Close and N. A. Tornqvist, J. Phys. G 28, R249 (2002).

[3] D. V. Bugg, Phys. Rep. 397, 257 (2004).

[4] C. Amsler and N. A. Tornqvist, Phys. Rep. 389, 61 (2004).

[5] R. L. Jaffe, Phys. Rep. 409, 1 (2005).

[6] E. Klempt and A. Zaitsev, Phys. Rep. 454, 1 (2007).

[7] N. N. Achasov, Phys. Usp. 41, 1149 (1998); Nucl. Phys. A675, 279 (2000).

[8] J. R. Peláez, Phys. Rep. 658, 1 (2016).

[9] N. N. Achasov and G. N. Shestakov, Phys. Usp. 62, 3 (2019).

[10] R. L. Jaffe, Phys. Rev. D 15, 267 (1977); 15, 281 (1977); G.'t Hooft, G. Isidori, L. Maiani, A. D. Polosa, and V. Riquer, Phys. Lett. B 662, 424 (2008); D. Ebert, R. N. Faustov, and V. O. Galkin, Eur. Phys. J. C 60, 273 (2009); G. Eichmann, C. Fischer, and W. Heupel, Phys. Lett. B 753, 282 (2016).

[11] J. D. Weinstein and N. Isgur, Phys. Rev. Lett. 48, 659 (1982); Phys. Rev. D 27, 588 (1983); 41, 2236 (1990); Yu. S. Kalashnikova and A. V. Nefediev, Phys. Usp. 62, 568 (2019).

[12] Z. G. Wang, Eur. Phys. J. C 76, 427 (2016).

[13] M. G. Alford and R. L. Jaffe, Nucl. Phys. B578, 367 (2000); H. Suganuma, K. Tsumura, N. Ishii, and F. Okiharu, Prog. Theor. Phys. Suppl. 168, 168 (2007); N. Mathur,
A. Alexandru, Y. Chen, S. J. Dong, T. Draper, I. Horváth, F. X. Lee, K. F. Liu, S. Tamhankar, and J. B. Zhang, Phys. Rev. D 76, 114505 (2007); M. Loan, Z. H. Luo, and Y. Y. Lam, Eur. Phys. J. C 57, 579 (2008); S. Prelovsek and D. Mohler, Phys. Rev. D 79, 014503 (2009); T. Kunihiro, S. Muroya, A. Nakamura, C. Nonaka, M. Sekiguchi, and H. Wada (SCALAR Collaboration), Phys. Rev. D 70, 034504 (2004).

[14] J. J. Dudek, R. G. Edwards, and D. J. Wilson (Hadron Spectrum Collaboration), Phys. Rev. D 93, 094506 (2016).

[15] E. van Beveren, T. A. Rijken, K. Metzger, C. Dullemond, G. Rupp, and J. E. Ribeiro, Z. Phys. C 30, 615 (1986).

[16] E. van Beveren and G. Rupp, Eur. Phys. J. C 22, 493 (2001).

[17] E. van Beveren, D. V. Bugg, F. Kleefeld, and G. Rupp, Phys. Lett. B 641, 265 (2006).

[18] N. A. Tornqvist and M. Roos, Phys. Rev. Lett. 76, 1575 (1996).

[19] N. A. Tornqvist, Z. Phys. C 68, 647 (1995); N. A. Tornqvist and M. Roos, Phys. Rev. Lett. 76, 1575 (1996).

[20] M. Boglione and M. R. Pennington, Phys. Rev. D 65, 114010 (2002).

[21] T. Wolkanowski, F. Giacosa, and D. H. Rischke, Phys. Rev. D 93, 014002 (2016).

[22] I. K. Hammer, C. Hanhart, and A. V. Nefediev, Eur. Phys. J. A 52, 330 (2016).

[23] J. Oller and E. Oset, Phys. Rev. D 60, 074023 (1999). 
[24] J. R. Pelaez and G. Rios, Phys. Rev. Lett. 97, 242002 (2006).

[25] J. Ruiz De Elvira, J. R. Pelaez, M. R. Pennington, and D. J. Wilson, Phys. Rev. D 84, 096006 (2011).

[26] J. Oller and E. Oset, Nucl. Phys. A620, 438 (1997).

[27] A. Dobado and J. Pelaez, Phys. Rev. D 56, 3057 (1997).

[28] A. Gomez Nicola and J. Pelaez, Phys. Rev. D 65, 054009 (2002).

[29] J. Pelaez, Phys. Rep. 658 (2016).

[30] J. R. Peláez and F. J. Yndurain, Phys. Rev. D 71, 074016 (2005); R. Garcia-Martin, R. Kaminski, J. R. Peláez, and J. Ruiz de Elvira, Phys. Rev. Lett. 107, 072001 (2011).

[31] J. R. Peláez, A. Rodas, and J. Ruiz De Elvira, Eur. Phys. J. C 79, 1008 (2019).

[32] R. Garcia-Martin, R. Kaminski, J. R. Peláez, J. Ruiz De Elvira, and F. J. Yndurain, Phys. Rev. D 83, 074004 (2011).

[33] A. M. Badalian, Phys. At. Nucl. 66, 1342 (2003).

[34] D. Darvish, R. Brett, J. Bulava et al., arXiv:1909.07747.

[35] A. Di Giacomo, H. G. Dosch, V. I. Shevchenko, and Yu. A. Simonov, Phys. Rep. 372, 319 (2002); Yu. A. Simonov, in QCD: Perturbative or Nonperturbative?, edited by L. S. Ferreira et al. (World Scientific, Singapore, 2001), p. 60.

[36] Yu. A. Simonov, Phys. Rev. D 99, 056012 (2019).

[37] Yu. A. Simonov, Phys. Rev. D 99, 096025 (2019).

[38] A. M. Badalian and B. L. G. Bakker, Phys. Rev. D 100, 034010 (2019).

[39] A. M. Badalian and B. L. G. Bakker, Phys. Rev. D 67, 071901 (2003); A. M. Badalian, B. L. G. Bakker, and Yu. A. Simonov, Phys. Rev. D 75, 116001 (2007); Yu. A. Simonov, Phys. Rev. D 88, 025028 (2013).

[40] E. Eichten, K. Gottfried, K. Kinoshita, K. D. Lane, and T.-M. Yan, Phys. Rev. D 17, 3090 (1978); 21, 203 (1980).
[41] E. Eichten, K. Lane, and C. Quigg, Phys. Rev. D 69, 094019 (2004); Yu. S. Kalashnikova, Phys. Rev. D 72, 034010 (2005)

[42] I. V. Danilkin and Yu. A. Simonov, Phys. Rev. D 81, 074027 (2010); Phys. Rev. Lett. 105, 102002 (2010); I. V. Danilkin, V. D. Orlovsky, and Yu. A. Simonov, Phys. Rev. D 85, 034012 (2012).

[43] Yu. A. Simonov, Phys. Rev. D 65, 094018 (2002); Phys. At. Nucl. 67, 846 (2004); 67, 1027 (2004); S. M. Fedorov and Yu. A. Simonov, JETP Lett. 78, 57 (2003).

[44] Yu. A. Simonov, Int. J. Mod. Phys. A 31, 1650104 (2016).

[45] J. Gasser and H. Leutwyler, Ann. Phys. (N.Y.) 158, 142 (1984); Nucl. Phys. B250, 465 (1985); B307, 763 (1988).

[46] Yu. A. Simonov, Phys. At. Nucl. 79, 265 (2016).

[47] A. Badalian, M. Lukashov, and Y. A. Simonov, arXiv: 2001.07113.

[48] Yu. A. Simonov, Phys. Rev. D 88, 025028 (2013).

[49] Yu. A. Simonov and J. A. Tjon, Ann. Phys. (N.Y.) 228, 1 (1993); 300, 54 (2002).

[50] A. Yu. Dubin, A. B. Kaidalov, and Yu. A. Simonov, Phys. Lett. B 343, 310 (1995).

[51] A. M. Badalian, Phys. At. Nucl. 63, 2173 (2000); A. M. Badalian and D. S. Kuzmenko, Phys. Rev. D 65, 016004 (2001); S. G. Gorishnii, A. L. Kataev, and S. A. Larin, Phys. Lett. 135B, 457 (1984); A. L. Kataev, Phys. At. Nucl. 68, 567 (2005).

[52] S. Godfrey and N. Isgur, Phys. Rev. D 32, 189 (1985).

[53] D. Ebert, R. N. Faustov, and V. O. Galkin, Phys. Rev. D 79, 114029 (2009).

[54] R. Giachetti and E. Sorace, Phys. Rev. D 87, 034021 (2013).

[55] F. Verstraete, V. Murg, and J. I. Cirac, Adv. Phys. 57, 143 (2008); R. Orus, Ann. Phys. (Amsterdam) 349, 117 (2014).

[56] A. M. Badalian (private communication). 